\title{
The false falx and tentorium sign: case report of subdural haematoma and sickle cells disease
}

\author{
Hernando Raphael Alvis-Miranda ${ }^{1}$, Carlos Fernando Lozano- \\ Tangua $^{2}$, Gabriel Alcala-Cerra ${ }^{2}$, Andres M. Rubiano ${ }^{3}$, Luis Rafael \\ Moscote-Salazar ${ }^{2}$ \\ ${ }^{1}$ Physician, Universidad de Cartagena \\ ${ }^{2}$ Neurosurgeon, Universidad de Cartagena \\ ${ }^{3}$ Neurosurgeon, Hospital Universitario de Neiva
}

\begin{abstract}
The increased density in the basal cisterns and the subarachnoid space on CT scans is a well-known characteristic of subarachnoid hemorrhage. Have been described diverse conditions that can emulate subarachnoid hemorrhage, such as purulent leptomeningitis, intrathecal contrast material and leak of high doses of intravenous contrast material to the subarachnoid space. We present the case of a male patient who presented a subdural hematoma in the setting of non-diagnosed sickle cell disease. To this patient was performed a panangiography which discard any aneurismal hemorrhage origin
\end{abstract}

Key words: subarachnoid hemorrhage, sickle cell disease, subdural hematoma, false falx and tentorium sign.

\section{Introduction}

Subarachnoid hemorrhage (SAH) appears characteristically in the CT scan as a hyperdensity in the basal cisterns and subarachnoid space (SAS). Diverse medical conditions can emulate SAH appearance, such as hypoxic encephalopathy, hyperperfusion radical encephalopathy, extensive infarctions, viral meningoencephalitis, purulent meningitis, bilateral subdural haematoma and idiopathic intracranial hypertension (1-6), but without clinical or anatomopathological hemorrhage findings(7), this phenomena is called "pseudo-SAH".

In 1980, Osborn (8) described the "false falx and tentorium sign" as a finding of pseudo-SAH. We present the clinical case of a patient with false falx and tentorium sign as a contribution to medical literature.

\section{CASE PRESENTATION}

Male patient, 75 years old, who presents to the ER of the Hospital Universitario del Caribe with right hip pain that hinders the gait during the last week and a fast declining conscious level. He has not important antecedents, no smoke, and no alcohol habit. At physical examination is evidenced drowsiness, bradypsyquia, time and space disorientation, 
Glasgow coma scale (GCS) 13/15 (ER: 3; VR: 4; MR: 6), obey orders, and has moderate neck stiffness. No motor or sensitive deficit at cranial nerves examination. CT scan is ordered, which evidences a left chronic laminar subdural hematoma without significant mass effect (Figure 1, A and B).

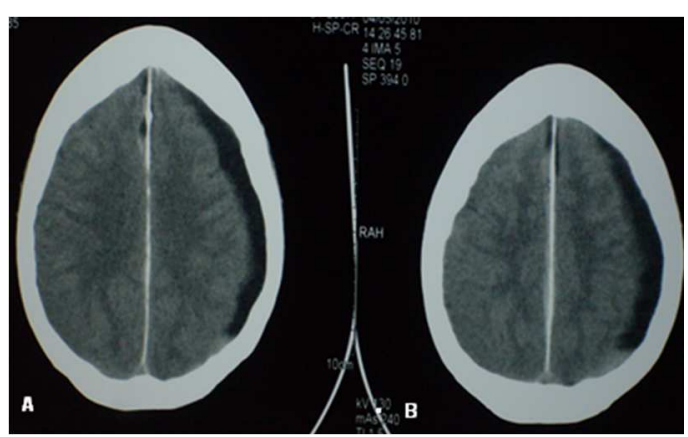

Figures 1, A and B - Non-contrasted brain CT-scan showing a left hemispherical subdural collection with mass effect

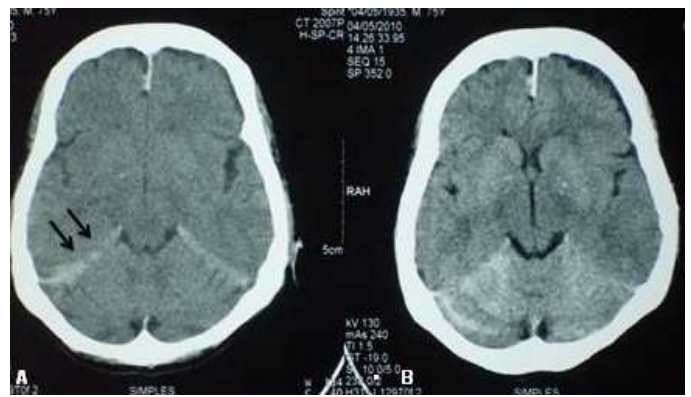

Figure 2, A and B - There is an interhemispherical fronto-parietal hemorrhagic collection, which extends toward the right side of the tentorium configuring SAH probably from pericallus artery bleeding

It is observed also a right frontoparietal interhemispheric hemorrhagic collection that extends to right tentorium cerebelli configuring a SAH presumably from pericallosal artery (Figure 2, A and B). The chronic subdural hematoma is considered an incidental finding. With a clinical impression of acute SAH the patient is hospitalized in ICU. The solicited laboratory test report: Hemoglobin: $10 \mathrm{~g} / \mathrm{dL}$, hematocrit: 34.1\%, platelets: $104.000 \mathrm{~mm} 3$, WBC: $10.900 \mathrm{~mm} 3$, neutrophils: $8.900 \mathrm{~mm} 3$.

Cerebral aneurismal disease is suspected, and is considered perform a cerebral panangiography previous application of three platelets units, and sickle solubility test, wich result positive, then hemoglobin electrophoresis is performed confirming the sickle cell disease. The cerebral panangiography rule out any aneurismal alteration, and are evidenced secondary changes to the bilateral collection on right frontoparietal convexity. (Figure 3, A and B).

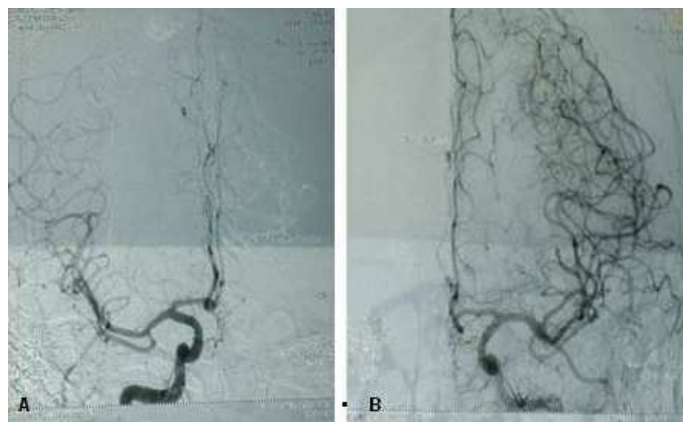

Figure 3, A and B - Cerebral panangiography, discarding vascular pathology

The patient has a temporal improvement of his neurological status. On the fourth day of admission appears diffuse abdominal pain, with prolonged control clotting times: PTT: 40 (control 28), mild conjunctival jaundice. Are requested liver function tests: AST: $122 \mathrm{mg} / \mathrm{dL}$ (normal value 42), ALT: $60 \mathrm{mg} / \mathrm{dL}$ (normal value 40), LDH: 422.7 (normal value 180), 
DOI: 10.2478/romneu-2014-0045

hemoglobin $8.3 \mathrm{~g} / \mathrm{dL}$ and hematocrit $25 \%$. The internal medicine evaluation report an acute liver failure in a patient GCS 15/15 and no neurological focalization. Fresh frozen plasma, compatible packed red blood cells and platelets are transfused. On the sixth day of admission, the patient develops right hemiparesis, dysphasia with motor predominance and deterioration of consciousness reaching coma. Despite polytransfusion patient develops pancytopenia. Bone marrow aspirate is performed on the left iliac crest showing no cellular infiltrate. There are no main changes on the simple CT scan respect the first.

Thus, it is considered that patient present sickle cell disease confirmed by hemoglobin electrophoresis, with transient ischemic brain events and infarctions that generate hip pain and acute progressive liver failure. Patient died eight days after admission, the cause of death is considered fulminant hepatic encephalopathy as the brain scan shows no changes respect to the first, where the neurological status is normal.

\section{Discusion}

SAH is a serious entity that requires early and precise diagnosis and treatment. The wide availability of brain CT scans allow accelerate the diagnosis and the detection of early complications, due to its high sensibility and specificity in the first 24 hours of the event. However, occasionally in the simple CT scans can be appreciated images simulating bleeding in the SAS, even without clinical or anatomopathological evidence of hemorrhage (9). This is a widely commented phenomenon in the medical literature, called "pseudo-SAH" (10). The pseudo-SAH hemorrhage in brain cisterns has received scarce attention in medical literature. However some authors had described weighted enhancement of falx cerebri and tentorium cerebelli in association with brain edema (11). This phenomenon has been described in cases of anoxic-ischemic encephalopathy with brain edema, bacterial meningitis, dural sinus thrombosis, brain metastasis, and as a complication of angiographic procedures, and even in healthy patients of all ages (11). Causes are described in the Box 1.

Spiegel et al (12) in 1989 were the first to note that in the brain edema secondary to encephalopathy, the CT scans images show a hyperdensity on basal cisterns and in the SAS, similar to that observed during acute SAH. They also reported that images of 10 patients with marked brain edema associated whether to tumor or to brain infarction, showed acute SAH appearance, especially in the course of interhemispheric fissure and tentorium cerebelli, posteriorly in the autopsy findings SAH was absent.

In 1998, Avrahmi et al (1), founded on the CT scans of 100 comatose patients with brain edema, similar findings of SAH along cisterns and grooves, discarding in all of them this diagnosis. They also suggest the term "pseudo" for this phenomenon.

\section{List of common causes of pseudo-SAH:}

- Cerebral gliomatosis

- Bilateral subdural hematoma

- Secondary polycythemia 
- High doses of contrast material used in spinal angiographic procedures

- Intrathecally administered contrast material

- Acute purulent leptomeningitis

- Intracranial idiopathic hypertension or hypotension

- Hipertensión o hipotensión intracraneal idiopática

- Viral meningoencephalitis

- Oters

In a simple CT scan of a healthy individual, skull bones are immediately delineated by a thin layer of duramater, and this in turn by the SAS, where is the cerebrospinal fluid (CSF). There is wide variability of mean SAS width, being greater in both life extremes.

In the particular case of brain edema secondary to hypoxic encephalopathy, cerebral cortex begins to move into areas normally occupied by LCR, allowing neighbor vein congestion, and grooves convexity disappearance, being hyperdense on the simple CT scans, simulating SAH (13). It is also envisaged that with severe brain ischemia the brain tissue density diminishes related to superficial cortex congestive veins, giving an impression of hyperdensity in the latter (14).

Contrasted CT scans can be useful to confirm the diagnosis of pseudo-SAH, because of the basal cisterns abnormal enhancement (15).

Other useful tool for distinguish SAH from Pseudo-SAH is the measurement of Houndsfield (HU) units in the hyperdense region (16). In the al-Yamani et al (17) study the mean value of accentuation quotients in the basal cisterns of patients with pseudo-SAH and spontaneous intracerebral hemorrhage was $29-33 \mathrm{HU}$, and in patients with aneurismal SAH was of $60-70 \mathrm{HU}$.

\section{Conclusions}

The importance of this case is to consider in the cases of suspected subarachnoid bleeding and increased density of the SAS, especially on the tentorium cerebelli and the falx cerebri, the presence of a false $\mathrm{SAH}$, corresponding to false falx and tentoium sign, which implies no aneurysmal rupture but forces us to discard with the complementary studies warranted in the vascular pathology evaluation.

\section{Correspondence \\ Dr. Luis Rafael Moscote. Neurosurgeon. Universidad de Cartagena, Cartagena de Indias, Colombia.E-mail: mineurocirujano@aol.com}

\section{References}

1. Avrahami E, Katz R, Rabin A, Friedman V. CT diagnosis of non-traumatic subarachnoid haemorrhage in patients with brain edema. Eur J Radiol 1998;28:222-5. 2. Given CA, Burdette JH, Elster AD, Williams DW. Pseudo-subarachnoid hemorrhage: a potential imaging pitfall associated with diffuse cerebral edema. AJNR Am J Neuroradiol 2003;24:254-6.

3. Cucchiara B, Sinson G, Kasner SE, Chalela JA. Pseudosubarachnoid hemorrhage: report of three cases and review of the literature. Neurocrit Care 2004;1:371-4.

4. You JS, Park S, Park YS, Chung SP. Pseudosubarachnoid hemorrhage. Am J Emerg Med 2008;26:521.e1-2.

5. Misra V, Hoque R, Gonzalez-Toledo E, Kelley RE, Minagar A. Pseudo-subarachnoid hemorrhage in a patient with acute cerebellar infarction. Neurol Res 2008;30:813-5.

6. Westwood AJ, Burns JD, Green DM. Teaching NeuroImages: Pseudo-subarachnoid hemorrhage. Neurology 2012;78:e54. 
DOI: 10.2478/romneu-2014-0045

7. De la Cruz-Cosme C, Barbieri G, Vallejo-Baez A. Pseudo-subarachnoid haemorrhage. A need for clinicalradiological diagnostic criteria. Neurología (English Edition) 2010;25:463-5.

8. Osborn AG, Anderson RE, Wing SD. The false falx sign. Radiology 1980;134:421-5.

9. Agha A, Al-Hakami M. A case report of pseudosubarachnoid hemorrhage. Maedica (Buchar) 2011;6:210-2.

10. Silberstein M. Diagnosis of pseudosubarachnoid hemorrhage. AJNR Am J Neuroradiol 2003;24:1492; author reply 1492 .

11. Eckel TS, Breiter SN, Monsein LH. Subarachnoid contrast enhancement after spinal angiography mimicking diffuse subarachnoid hemorrhage. AJR Am J Roentgenol 1998;170:503-5.

12. Spiegel SM, Fox AJ, Vinuela F, Pelz DM. Increased density of tentorium and falx: a false positive CT sign of subarachnoid hemorrhage. Can Assoc Radiol J 1986;37:243-7.

13. Thomas GL, Stachowski ER. Pseudosubarachnoid haemorrhage on CT brain scan: an unusual presentation of diffuse hypoxic brain injury. Intensive Care Med 2007;33:2038-40.

14. Schievink WI, Wijdicks EF, Meyer FB, Sonntag VK. Spontaneous intracranial hypotension mimicking aneurysmal subarachnoid hemorrhage. Neurosurgery 2001;48:513-6; discussion 516-7.

15. Gutierrez LG, Rovira A, Portela LAP, Leite C da C, Lucato LT. CT and MR in non-neonatal hypoxicischemic encephalopathy: radiological findings with pathophysiological correlations. Neuroradiology 2010;52:949-76.

16. Senthilkumaran S, Balamurugan N, Menezes RG, Thirumalaikolundusubramanian P. Role of Hounsfield units to distinguish pseudo-subarachnoid hemorrhage. Clin Toxicol (Phila) 2011;49:948.

17. al-Yamany M, Deck J, Bernstein M. Pseudosubarachnoid hemorrhage: a rare neuroimaging pitfall. The Canadian Journal of Neurological Sciences. Le Journal Canadien Des Sciences Neurologiques 1999;26:57-9. 